\title{
Influência da Preservação de Pele em Cirurgiala Conservadora por Câncer de Mama sobre as Taxas de Recorrência Local e a Distância
}

\author{
Influence of Skin Sparing in Conservative Surgery for Breast \\ Cancer on the Rates of Local and Distant Recurrence
}

Marcos Desidério Ricci ${ }^{1}$, José Aristodemo Pinotti ${ }^{1}$, Filomena Marino Carvalho², Marianne Pinotti ${ }^{1}$, Arícia Helena G. Giribela ${ }^{1}$

\begin{abstract}
RESUM0
Objetivo: avaliar a taxa de recorrência local, regional e a distância de nova técnica cirúrgica para tratamento conservador do câncer de mama de estádio inicial. A técnica é baseada na ressecção segmentar com dissecção axilar e preservação de pele por incisão periareolar única.

Métodos: cento e dezenove pacientes com câncer de mama de estádio I e II foram incluídas neste estudo. O grupo estudo foi constituído por 57 pacientes que se submeteram à cirurgia pela técnica proposta e 62 pacientes submetidas a quadrantectomia clássica constituíram o grupo controle. A radioterapia pós-operatória e oboost foram realizados em ambos os grupos. A média de seguimento foi de 50,1 meses para o grupo estudo e 51,2 para o grupo controle. As taxas de recidiva, sobrevida global e sobrevida livre de doença foram analisadas $e$ comparadas entre os dois grupos.

Resultados: a taxa de recidiva local no periodo foi de 3,5\% para o grupo estudo e 4,8\% para o grupo controle. Não houve diferença significativa entre os grupos nas taxas de sobrevida livre de doença e sobrevida global.

Conclusões: a técnica de ressecção segmentar com dissecção axilar por incisão única periareolar, preservando pele, não mostrou diferença, em relação à quadrantectomia clássica, nas taxas de recidiva, sobrevida global e sobrevida livre de doença.
\end{abstract}

PALAVRAS-CHAVE: Mama: câncer. Cirurgia conservadora.Câncer: prognóstico.

Introdução

Na segunda metade do século vinte, o tratamento cirúrgico conservador do câncer de mama foi tema constante de publicações científicas. Apesar disso, a mastectomia radical à Halsted foi a técnica mais utilizada em tumores de qualquer estádio clínico. As publicações de Veronesi et al. ${ }^{1}$, Fisher et al. $^{2}$, e o Consensus Development Conference

${ }^{1}$ Departamento de Ginecologia e Obstetrícia da Faculdade de Medicina da Universidade de São Paulo (FMUSP),

${ }^{2}$ Departamento de Patologia da FMUSP

Correspondência :

Marcos Desidério Ricci

Rua Ministro Godoi, $\mathrm{n}^{\circ} 432$ - Perdizes

05015-000 - São Paulo - SP

Tel: (011) 3862-2294 - FAX: (011) 3872-0923

e-mail: desiderion@uol.com.br
Statement (1990) ${ }^{3}$, organizado pelos National Institutes of Health, foram passos marcantes na efetivação da cirurgia conservadora como tratamento oncologicamente apropriado para a maioria das mulheres com câncer de mama de estádio inicial. Recentes linhas de pesquisa procuram definir o papel da preservação dos linfonodos axilares com base na biópsia do linfonodo sentinela e da limitação da ressecção mamária pela ablação do tumor guiada por métodos de imagem, e a simplificação do tratamento radioterápico tem sido obtida por meio da braquiterapia intersticial e feixe de elétrons intra-operatório ${ }^{4}$.

O controle local do tumor, por meio da radioterapia adjuvante e análise das margens do espécime cirúrgico são condições que devem acompanhar as ressecções mamárias reduzidas. As recidivas locais têm relação estreita com o estado das 
margens cirúrgicas, a presença de componente intraductal extenso, embolização peritumoral vascular, tipo histológico lobular, grau nuclear e tamanho tumoral ${ }^{5}$. A extensão da ressecção ideal do tecido mamário sofre influência das características do tumor e do tecido mamário circunjacente, modificadas pela idade da paciente, estado hormonal e densidade mamária.

As divergências existentes na literatura, quanto às diversas técnicas de cirurgia conservadora, motivaram este estudo. A proposta da técnica de ressecção segmentar mamária com preservação de pele e dissecção axilar por incisão única periareolar foi descrita em 1992. Os resultados preliminares desta técnica, quanto aos resultados estéticos, foram publicados recentemente ${ }^{5}$. Os autores avaliam neste trabalho a influência da preservação de pele sobre as taxas de recidiva local, regional e a distância, nas pacientes submetidas à cirurgia conservadora por câncer de mama no estádio inicial.

\section{Pacientes e Métodos}

No período de junho de 1992 a outubro de 2001, 119 pacientes portadoras de carcinoma mamário invasor, estadiadas como T1 ou T2, e NO ou N1 (TNM/UICC), foram selecionadas para o estudo na Clínica Prof. José Aristodemo Pinotti, Hospital das Clínicas da Faculdade de Medicina da Universidade de São Paulo, e Centro de Referência da Saúde da Mulher. Foram constituídos dois grupos, não randomizados, cuja seleção se baseou na distância do tumor à pele, avaliada por critérios ultra-sonográficos e radiológicos. Não houve diferenças ecográficas dos nódulos entre os grupos, de acordo com os critérios de malignidade de Stavros et al. ${ }^{6}$. As pacientes com tumor distante mais de $2 \mathrm{~cm}$ da superfície cutânea formaram o grupo estudo (GEstudo) e aquelas com distância menor constituíram o grupo controle (GControle). As taxas de recidiva local, regional e metástase à distância foram registradas, bem como as curvas de sobrevida global e sobrevida livre de doença.

A recidiva local foi dita verdadeira quando presente no local do espécime do quadrante retirado ou em área adjacente, e não verdadeira, quando localizada em região distinta e afastada da área do tumor primário em pelo menos 3 centímetros.

No GEstudo, as pacientes foram tratadas por cirurgia pela técnica de ressecção segmentar com dissecção axilar por incisão única periareolar, e no GControle, pela técnica de quadrantectomia clássica com dissecção axilar pela mesma incisão. A análise intra-operatória de verificação das margens livres, pela técnica do imprint, foi aplicada nas pacientes pertencentes aos dois grupos ${ }^{5}$. Esta prática determinou a mudança de procedimento no intra-operatório em duas pacientes do GEstudo e uma do GControle, abortando a técnica em estudo e substituindo-a pela mastectomia glandular subcutânea com dissecção axilar. A razão que motivou a ampliação da ressecção mamária nestes casos foi a presença do carcinoma ductal in situ com extenso componente intracanalicular.

$\mathrm{Na}$ maioria das vezes, o esclarecimento histológico de malignidade da lesão foi feito no préoperatório por biópsia com agulha grossa guiada por ultra-sonografia (core biopsy).

\section{Grupo de Estudo}

A idade das pacientes variou de 31 a 77 anos, com média de 51,7 anos e mediana de 50 anos. O tempo de seguimento mínimo foi de 13 meses, máximo de 111 meses, média de 50,1 meses e mediana de 46 meses. Em relação ao estadiamento clínico (TNM/UICC, 1997), 41 pacientes $(71,9 \%)$ estavam no estádio I no momento do diagnóstico, 14 pacientes $(24,5 \%)$ no estádio IIA e duas pacientes $(3,5 \%)$ no estádio IIB. Os tipos histológicos encontrados foram os seguintes: carcinoma ductal invasivo, em 53 pacientes $(92,9 \%)$; carcinoma tubular, em duas (3,5\%); carcinoma túbulo-lobular, em uma $(1,7 \%)$, e carcinoma colóide, em uma (1,7\%).

As pacientes selecionadas não demonstravam evidência clínica, radiológica ou ecográfica de envolvimento cutâneo. A distância do tumor à pele foi de cerca de $2 \mathrm{~cm}$, permitindo, desta forma, manter a margem de segurança necessária. Esta distância foi analisada pela interpretação da mamografia convencional na incidência crâniocaudal e/ou perfil. Os tumores de localização central, retroareolares, aqueles com suspeita de serem multicêntricos ou multifocais e os carcinomas lobulares foram excluídos deste grupo.

A marcação cutânea da projeção do tumor na pele e da área a ser ressecada foi feita com caneta dermográfica. A incisão periareolar incluindo pele e tecido celular subcutâneo foi feita com bisturi e incluiu até $180^{\circ}$ de sua circunferência. O espécime a ser retirado incluiu a região do tumor envolto por parênquima mamário, que contempla em média $2 \mathrm{~cm}$ até as margens de ressecção e a fáscia do músculo peitoral maior, preservando pele e gordura subdérmica.

As margens do espécime foram marcadas com fio de algodão ou placas metálicas específicas, a fim de servir como orientação tridimensional no momento da análise cito-histológica das margens.

O cirurgião descolou com tesoura ou bisturi elétrico a porção da glândula mamária no seu plano profundo em direção à borda lateral do músculo 
peitoral maior. Nesse instante, a fáscia ao longo da borda lateral deste músculo foi seccionada e o auxiliar colocou uma válvula de Doyen na sua margem profunda, elevando-o por tração para que o cirurgião pudesse promover a dissecção dos linfonodos interpeitorais dispostos entre este músculo e o peitoral menor (cadeia de Rotter). Na borda lateral do músculo peitoral menor a fáscia clavicotoracopeitoral foi apresentada e incisada, expondo a cavidade axilar.

Uma válvula de Doyen, disposta sob o músculo peitoral maior, o elevou de forma a apresentar para o cirurgião a cavidade axilar. O cirurgião promoveu então a dissecção no sentido médio-lateral e crânio-caudal sobre o conjunto linfoadiposo do nível III para o nível II de Berg, na topografia da veia axilar, com a finalidade de promover a exposição desta, bem como de suas colaterais ${ }^{7}$.

A dissecção axilar em monobloco prosseguiu com pinça e tesoura de Metzenbaum longa, realizada no sentido do nível III para o nível I, reconhecendo-se o feixe vásculo-nervoso toráco-acromial e a veia mamária externa. Reconhecemos e preservamos o nervo torácico longo e o feixe vásculo-nervoso toracodorsal. O nervo intercostobraquial, que emerge da parede torácica, atravessa a cavidade axilar e penetra no retalho cutâneo lateral, foi preservado sempre que as condições técnicas assim o permitiram. Caso a área de ressecção mamária fosse volumosa a ponto de promover defeito estético visivelmente insatisfatório, pôde-se dispor de porções adjacentes da glândula mamária, que foram descoladas em direção à fáscia muscular ou ao tecido subcutâneo para preencher a área cruenta. Em seguida colocamos um dreno de vácuo-aspiração com sistema fechado compreendendo a mama e cavidade axilar. Realizamos então a sintese da pele da borda areolar com fio de náilon 4-0.

\section{Grupo Controle}

A idade das pacientes variou de 26 a 74 anos, com média de 49,1 anos, e mediana de 49 anos. O tempo de seguimento mínimo foi de 12 meses, máximo de 120 meses, média de 51,2 meses e mediana de 49 meses. Em relação ao estadiamento clínico (TNM/UICC, 1997), 28 pacientes $(45,1 \%)$ pertenciam ao estádio I no momento do diagnóstico; $32(51,6 \%)$ ao estádio IIA e duas pacientes $(3,2 \%)$ ao estádio IIB. Os tipos histológicos encontrados foram os seguintes: carcinoma ductal invasivo, em 61 pacientes $(98,4 \%)$, e carcinoma mucinoso, em uma paciente $(1,6 \%)$.

A técnica operatória do GControle diferiu daquela usada no GEstudo apenas em relação à tática da ressecção mamária. Os tempos correspondentes à linfadenectomia axilar foram semelhantes em técnica e seqüência.
A incisão da pele e tecido celular subcutâneo foi feita com bisturi de lâmina fria, garantindo margens laterais de 1 a $2 \mathrm{~cm}$. O formato da incisão variou de acordo com a localização topográfica do tumor na mama e a proposta de reparo estético previamente discutida com o cirurgião plástico. A seguir foi feita a diérese das margens laterais do espécime, constituído por segmento de pele e parênquima mamário, com margem de $2 \mathrm{~cm}$ de tecido em torno da área tumoral. O plano profundo de dissecção incluiu a fáscia do músculo peitoral maior. Os dois grupos de pacientes receberam a mesma rotina de tratamento complementar.

A irradiação compreendeu todo o volume mamário, utilizando equipamentos de megavoltagem (cobaltorerapia ou aceleradores lineares de 4 a 6 MV), até a dose de 4500 a 5000 cGy num período de 4 a 5 semanas. As pacientes com linfonodos axilares comprometidos por metástase tiveram o campo de irradiação, bem como a dose, estendido para a fossa supraclavicular. O boost, como reforço da dose de irradiação, foi feito no leito tumoral utilizando radiação $\mathrm{X}$ ou gama, braquiterapia ou elétrons em dose de 1000 a 1500 cGy. A irradiação mamária seguiu as orientações da International Commission on Radiological Protection (ICRP) ${ }^{8}$. O início da radioterapia ocorreu nos seis primeiros meses após a cirurgia.

A quimioterapia neoadjuvante foi realizada em nove pacientes $(15,8 \%)$ do GEstudo e oito pacientes $(12,9 \%)$ do GControle. Estas pacientes foram estadiadas clinicamente como IIA ou IIB, e participaram de um protocolo institucional do Serviço de Mastologia do Centro de Referência da Saúde da Mulher e do Hospital das Clínicas da Universidade de São Paulo. Os esquemas oferecidos foram o FEC, com 5-fluoracil (500 mg/m²), epirrubicina (50 mg/ $\mathrm{m}^{2}$ ) e ciclofosfamida $\left(500 \mathrm{mg} / \mathrm{m}^{2}\right)$, ou o $\mathrm{AC}$, com adriamicina (50 mg/ $\mathrm{m}^{2}$ ) e ciclofosfamida (500 mg/ $\mathrm{m}^{2}$ ). A quimioterapia constou de três ciclos com intervalo de 21 dias em média. A quimioterapia adjuvante foi realizada em 36 pacientes $(63,1 \%)$ do GEstudo e em $54(87,1 \%)$ do GControle. As pacientes que não receberam tratamento quimioterápico tinham idade superior a 70 anos e contra-indicações clínicas, ou tinham axila negativa e tumores com baixo risco de recidiva.

A hormonioterapia, prescrita por dois a cinco anos com tamoxifeno (20 mg/dia), foi iniciada após a quimioterapia nas pacientes com receptores estrogênicos positivos na pré e pós-menopausa.

As orientações do Conselho Nacional de Saúde, sobre "Ética em Pesquisa", foram seguidas, no momento do início da pesquisa, com base na Resolução CNS 01/88, publicada em 1988. As modificações pertinentes foram feitas e aplicadas, a partir de 1996, com base na Resolução CNS 196/96 - "Di- 
retrizes e Normas Regulamentadoras de Pesquisas envolvendo Seres Humanos", que revogou a resolução anterior ${ }^{9}$.

Durante todas as fases da pesquisa e compilação dos dados, foram seguidas as normas editadas pelo Código de Ética Médica ${ }^{10}$ estando de acordo com os artigos versados no capítulo XII (Pesquisa Médica) e capítulo XIII (Publicidade e Trabalhos Científicos).

A obtenção do consentimento livre e informado foi realizada pelo pesquisador principal e pelos demais médicos que participaram da pesquisa, durante a consulta pré-operatória.

As variáveis classificatórias foram apresentadas descritivamente em tabelas contendo freqüências absolutas e relativas (\%). Para comparação entre os grupos utilizou-se o teste $\chi^{2}$ ou teste da razão de verossimilhança ou teste exato de Fisher.

As variáveis quantitativas foram apresentadas em tabelas contendo médias, desvios padrão, mediana, valores mínimos e máximos. Os grupos foram comparados com teste $t$ de Student ou teste da soma de postos de Wilcoxon. Para as curvas de sobrevivência, utilizou-se o método de KaplanMeier, e para a comparação, o teste log-rank. Os valores de $\mathrm{p}<0,05$ foram considerados significantes.

\section{Resultados}

No GEstudo, das 57 pacientes, duas desenvolveram recidivas locais $(3,5 \%)$. Uma delas foi verdadeira e a outra não verdadeira, nenhuma delas na pele. A paciente com recidiva local verdadeira, diagnosticada 41 meses após a cirurgia, apresentava por tipo histológico o carcinoma túbulo-lobular e estádio patológico T1b NO. Uma recidiva regional em linfonodo axilar havia sido diagnosticada previamente no $19^{\circ}$ mês após a cirurgia.

A outra paciente do GEstudo apresentou recidiva não verdadeira, diagnosticada 40 meses após a cirurgia. O estádio patológico prévio foi Tla NO, e o tipo histológico, o carcinoma ductal invasivo com componente intraductal.

No GControle, das 62 pacientes, três desenvolveram recidiva local $(4,8 \%)$. Duas foram consideradas recidivas verdadeiras, com diagnóstico feito no local do tumor primário. Não houve recidiva na pele. O parênquima mamário foi envolvido pela recidiva tumoral nos três casos, e a fáscia e músculo peitoral maior em dois deles. O tempo transcorrido entre a cirurgia e a recidiva foi de $12,34 \mathrm{e}$ 98 meses. Os dados principais e alguns fatores de risco de recidiva local encontram-se na Tabela 1.

Tabela 1 - Características clínicas e patológicas e tratamento adjuvante das pacientes que apresentaram recidiva local no GEstudo e GControle.

\begin{tabular}{|c|c|c|c|c|c|c|c|c|c|}
\hline & Idade & TNM patológico & Tipo histológico & Grau histológico & $\mathrm{Tu}(\mathrm{mm})$ & CDIS & Linfonodos & $\mathrm{RE}$ & QT \\
\hline \multicolumn{10}{|l|}{ GEstudo } \\
\hline Caso 25 & 38 & T1aN0 & CDI & ॥ & 5 & + & $(0 / 29)$ & pos. & não \\
\hline Caso 46 & 66 & T1bN0 & C. Túbulo-lobular & I & 9 & - & $(0 / 13)$ & pos. & não \\
\hline \multicolumn{10}{|l|}{ GControle } \\
\hline Caso 3 & 45 & T1cN1 & $\mathrm{CDI}$ & III & 15 & - & $(2 / 8)$ & pos. & $\operatorname{sim}$ \\
\hline Caso 9 & 35 & T2NO & CDI & III & 21 & + & $(0 / 14)$ & neg. & $\operatorname{sim}$ \\
\hline Caso 15 & 49 & T1cN0 & CDI & III & 11 & - & $(0 / 24)$ & pos. & $\operatorname{sim}$ \\
\hline
\end{tabular}

$\mathrm{CDI}$ = carcinoma ductal invasivo; $\mathrm{CDIS}$ = carcinoma ductal in situ; $\mathrm{C}$. túbulo-lobular = carcinoma túbulo-lobular; $\mathrm{RE}$ = receptores estrogênicos; $\mathrm{QT}$ = quimioterapia; pres = presente; pos = positivo; neg = negativo; $\mathrm{Tu}=$ tumor.

A avaliação feita pelo teste $\chi^{2}$ entre as taxas de recidiva local registradas nas pacientes do GEstudo e GControle $(\mathrm{p}=0,507)$ não mostrou diferença significativa (Tabela 2). Não houve diferença significativa entre as taxas de recidiva regional de ambos os grupos, avaliadas pelo teste $\chi^{2}$, com $\mathrm{p}=0,180$.

Tabela 2 - Taxas de recidiva local entre pacientes do Gestudo e GControle $(p=0,507)$.

\begin{tabular}{crrrrrr}
\hline Recidiva local & \multicolumn{2}{c}{ GEstudo } & \multicolumn{2}{c}{ GControle } & \multicolumn{2}{c}{ Total } \\
\hline Não & 55 & $96,4 \%$ & 59 & $95,1 \%$ & 114 & $95,8 \%$ \\
Sim & 2 & $3,5 \%$ & 3 & $4,8 \%$ & 5 & $4,2 \%$ \\
Total & 57 & $100 \%$ & 62 & $100 \%$ & 119 & $100 \%$ \\
\hline
\end{tabular}

Metástases a distância foram observadas em cinco casos do GEstudo e em nove do GControle. Não houve diferença significativa entre os grupos quanto à taxa de metástase a distância avaliada pelo teste $\chi^{2}$, com $\mathrm{p}=0,206$ (Tabela 3 ). As curvas de sobrevida global, construídas com base no método de Kaplan-Meier e censuradas em 111 meses, não mostraram diferenças estatisticamente significativas entre os dois grupos, comparados pelo teste log-rank, com p=0,7550 (Figura 1). As curvas de sobrevida livre de doença dos grupos estudo e controle, construídas pelo método de Kaplan-Meier com censura em 111 meses, e comparadas pelo teste log-rank, com $\mathrm{p}=0,1960$, também não mostraram diferenças significativas (Figura 2). 


\begin{tabular}{|c|c|c|c|c|c|c|}
\hline \multirow{2}{*}{$\begin{array}{c}\begin{array}{c}\text { Metástase a } \\
\text { distância }\end{array} \\
\text { Não }\end{array}$} & \multicolumn{2}{|c|}{ GEstudo } & \multicolumn{2}{|c|}{ GControle } & \multicolumn{2}{|c|}{ Total } \\
\hline & 52 & $91,2 \%$ & 53 & $85,4 \%$ & 105 & $88,2 \%$ \\
\hline Sim & 5 & $8,7 \%$ & 9 & $14,5 \%$ & 14 & $11,7 \%$ \\
\hline Total & 57 & $100 \%$ & 62 & $100 \%$ & 119 & $100 \%$ \\
\hline
\end{tabular}

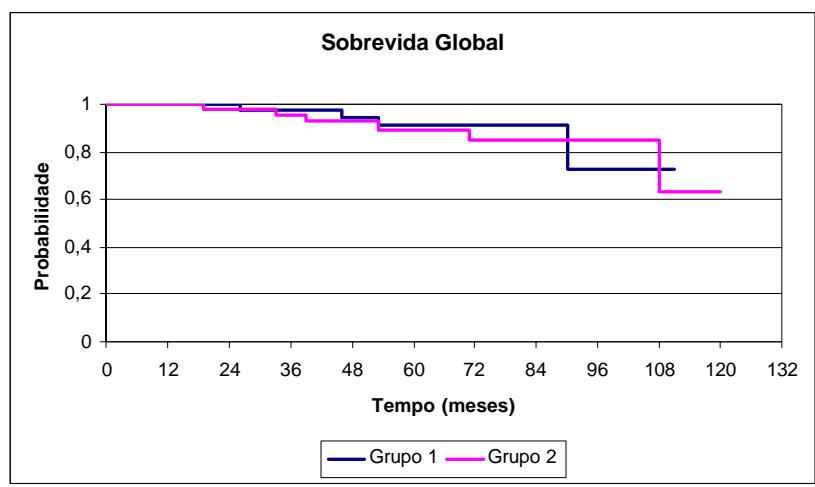

Figura 1 - Curva de sobrevida global entre pacientes do grupo GEstudo e GControle. $\mathrm{p}=0,7550$ (teste log-rank).

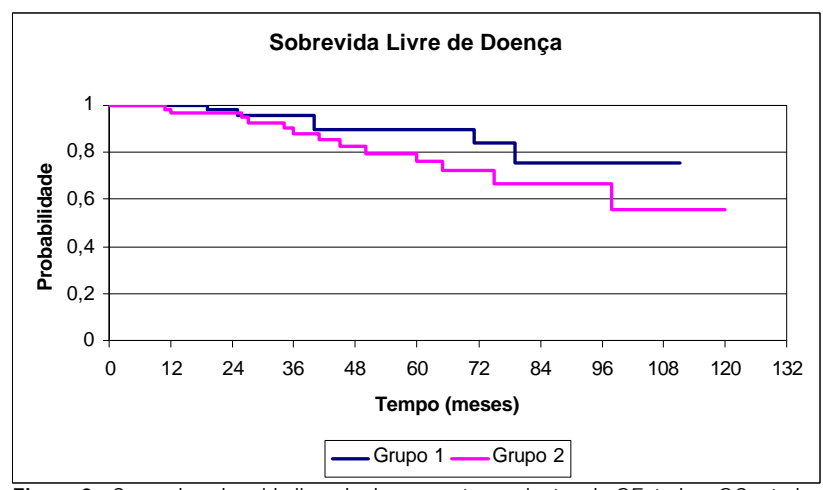

Figura 2 - Curva de sobrevida livre de doença entre pacientes do GEstudo e GControle. $\mathrm{p}=0,1962$ (teste log-rank).

\section{Discussão}

Não houve diferença significante entre as pacientes pertencentes ao GEstudo e GControle, quanto às taxas de sobrevida global e sobrevida livre de doença durante o período de seguimento analisado.

A técnica cirúrgica apresentada neste trabalho difere da quadrantectomia clássica por preservar pele, e da tumorectomia ou lumpectomia por não ser a incisão cutânea feita topograficamente sobre o tumor, mas periareolar. Veronesi et al. ${ }^{11}$, após 10 anos de seguimento de pacientes randomizadas a submeterem-se a QUART ou TART por tumores de até $2,5 \mathrm{~cm}$, observaram 9,3\% e 23\% de recidiva local, respectivamente. A diferença destas taxas pode ter, como uma das explicações, o fato de a tumorectomia realizada se basear na excisão do tumor com margem em torno de $10 \mathrm{~mm}$, enquanto na quadrantectomia a margem era de cerca de $20 \mathrm{~mm}$. A ressecção segmentar periareolar, proposta neste trabalho, difere da TART porque o controle das margens foi monitorado por parâmetros radiológicos, citológicos e histológicos.

A preocupação com a segurança oncológica desta técnica foi registrada pelo impacto da conservação de pele sobre o controle local da neoplasia. A comparação das taxas de recidiva local nas pacientes do GEstudo e GControle não mostrou diferença significante no período analisado.

A preservação de pele e limitação da ressecção tecidual foram garantidas pela monitorização intraoperatória das margens cirúrgicas, que permitiu identificar neste momento fatores promotores de maior recidiva local. A pele ao ser poupada contribui substancialmente na melhora do resultado estético, embora seja controverso seu efeito sobre as taxas de recidiva local.

Kurtz et al. ${ }^{12}$ descreveram 11 casos $(0,7 \%)$ de recidiva local na pele, dentre 1593 pacientes submetidas à cirurgia conservadora seguida da radioterapia. A recorrência foi associada à presença de fatores prognósticos desfavoráveis, incluindo metástase para linfonodos axilares, grau histológico III, receptor estrogênico negativo, invasão vascular e linfática. As pacientes com recidiva local tiveram pior prognóstico, que foi relacionado com doença primária agressiva biologicamente e taxa global de sobrevida menor.

Arcangeli et al. ${ }^{13}$, comparando a QUART e a TART quanto à recidiva local em 275 pacientes com tumores T1 ou T2 (TNM/UICC), seguidas por 58 meses em média, encontraram taxas de recidiva local de 4,9\% para a técnica TART e 5,9\% para a QUART. Nesta série, de Arcangeli et al. ${ }^{13}$, a indicação da cirurgia conservadora se baseou na exclusão de pacientes com carcinoma ductal in situ com componente intraductal extenso. Além disso, todas as pacientes foram submetidas a reforço da dose de radioterapia boost.

Gage et al. ${ }^{14}$ reviram 1.624 pacientes com câncer de mama estádio clínico I ou II tratadas por cirurgia conservadora e radioterapia pós-operatória no Joint Center for Radiation Therapy (Boston, MA). O boost foi efetuado nas pacientes com comprometimento das margens cirúrgicas. Os autores registraram a freqüência e os efeitos da recidiva local na pele sobre a recorrência a distância, com seguimento médio de 137 meses. A recidiva local no parênquima mamário ocorreu em 229 pacientes $(8 \%)$, ao passo que a recidiva em pele se deu em apenas 18 pacientes $(1,1 \%)$. Embora rara, a recidiva na pele foi associada a taxas 
significativamente elevadas, de 44\%, de metástases a distância no momento do seu diagnóstico, ou nos dois meses seguintes. Por outro lado, esta associação com a recidiva local no parênquima mamário foi de apenas 5\%. Estes autores também verificaram uma estreita relação entre recidiva local e presença de tumores biologicamente agressivos no momento do diagnóstico primário. A influência da preservação de pele sobre a taxa de recidiva local foi relatada pelo NSABP B-06, que registrou a recorrência limitada à pele e papila em apenas seis casos $(0,5 \%)$, dentre 1.108 pacientes submetidas a lumpectomia ${ }^{15}$. Segundo esse mesmo estudo, a remoção de pele não foi vista como fundamental na melhora do controle local, por se basear em um princípio não confirmado da cirurgia halstediana, porque o resultado estético fica comprometido, e finalmente porque aquelas pacientes com tumores que têm envolvimento cutâneo não são fortes candidatas a conservação da mama.

Das 119 pacientes operadas neste estudo, representando o GEstudo e GControle, e seguidas por cerca de 50 meses em média, se registrou a recidiva local em apenas cinco pacientes $(4,2 \%)$. Nenhuma delas foi cutânea, sendo verdadeiras apenas três recidivas $(2,5 \%)$.

A tática de ressecar a glândula mamária e preservar pele, visando à manutenção do envelope cutâneo para preenchimento por implante de silicone ou retalho desepitelizado, tem sido motivo de diversas publicações. A mastectomia subcutânea e a mastectomia com preservação de pele, denominada skin-sparing mastectomy, têm servido a esse propósito ${ }^{16-22}$. A mastectomia subcutânea e a mastectomia com preservação de pele (skin-sparing mastectomy) assumem semelhanças com a técnica discutida nesse trabalho à medida que o leito tumoral acaba por ficar revestido, na maioria das vezes, pelo retalho cutâneo original. Singletary e $\mathrm{Kroll}^{16}$, no M.D. Anderson Cancer Center, tiveram taxa de RL de 2,6\%, em 545 pacientes com câncer de mama estádio I e II submetidas a skin-sparing mastectomy, seguidas por quatro anos ${ }^{16}$.

Slavin et al. ${ }^{17}$ estudaram 51 pacientes com câncer de mama em estádio inicial, submetidas a skin-sparing mastectomy com reconstrução primária por retalho miocutâneo do músculo grande dorsal e prótese salina, entre 1991 a 1994. De forma original, após a mastectomia estes autores efetuaram aleatoriamente, em 32 pacientes, 114 biópsias no retalho cutâneo adjacente ao sítio tumoral. O exame anatomopatológico destas amostras não revelou ductos mamários na derme. Todas as pacientes foram seguidas em média por 44,8 meses. Ocorreram dois casos de recidiva local, sendo uma na pele $(1,9 \%)$ e outra no músculo peitoral maior $(1,9 \%)$.
Toth et al. ${ }^{18}$ registraram as taxas de recidiva local, metástase a distância e morte, em 50 pacientes com câncer de mama estádio I, II ou III que sofreram skin-sparing mastectomy e reconstrução imediata. Estas foram seguidas em média por 51,1 meses. Houve no período quatro casos de metástase óssea e um de metástase cerebral. Não foi registrada nenhuma recidiva local. Simmons et al. ${ }^{19}$ randomizaram 231 pacientes com câncer de mama de estádio inicial a se submeterem a mastectomia total (grupo I) ou skin-sparing mastectomy (grupo II), ambas com reconstrução imediata. Em 5 anos de seguimento as taxas de recidiva local não demonstraram diferença significativa, sendo de 3,2\% para o grupo I e 3,9\% para o grupo II. Estes autores têm incluído a skin-sparing mastectomy como técnica alternativa, oncologicamente segura, para tratamento de pacientes com câncer de mama de estádio inicial. Os resultados habitualmente demonstram minima deformidade, produzindo bom resultado estético, relacionado com a preservação da pele que envolve a mama.

A preservação da pele é exeqüivel e segura quando associamos dados clínicos e radiológicos à análise anatomopatológica intra-operatória. A técnica de avaliação intra-operatória das margens cirúrgicas, aplicadas em todos os casos do grupo I e II, demonstrou, numa análise inicial com seguimento médio de 40,6 meses, apenas um caso de recidiva local dentre 102 pacientes submetidas à cirurgia conservadora mais radioterapia pós-operatória por câncer de mama com diâmetro de até $5 \mathrm{~cm}^{23}$.

Com base em nossos resultados, da preservação de pele nas cirurgias conservadoras por câncer de mama, reforçado pelos relatos de literatura, podemos concluir que não há diferenças quanto às taxas de recidiva local, regional e a distância, em relação às pacientes que sofrem a ressecção cutânea.

\section{ABSTRACT}

Purpose: to evaluate the local, regional and distant recurrence rate of a new surgical technique for the conservative treatment of the breast cancer in early stages. The technique is based on breast segmental resection with axillary dissection and skin sparing by a single periareolar incision.

Methods: one hundred and nineteen patients with breast cancer stages I and II constituted the present study. The study group comprised fifty-seven patients who were submitted to surgery by the proposed technique, while 62 patients submitted to the classic quadrantectomy constituted the control group. Postoperative radiotherapy and boost were perfomed in both groups. The average follow-up was 50.1 
months for the study group and 51.2 months for the control group. The rates of recurrence, global survival and diseasefree survival were analyzed and compared between the two groups.

Results: the rate of local recurrence in the period was $3.5 \%$ for the study group and $4.8 \%$ for the control group. There was no statistically significant difference between the groups as to disease-free survival and global survival rates.

Conclusions: we demonstrated that the technique of segmental resection with axillary dissection by a single periareolar incision, preserving skin, did not show difference in relation to the classic quadrantectomy, regarding rates of recurrence, global survival and disease-free survival.

KEYWORDS: Breast cancer. Conservative surgery. Cancer: prognosis.

\section{Referências}

1. Veronesi U, Saccozzi R, Del Vecchio M, et al. Comparing radical mastectomy with quadrantectomy, axillary dissection, and radiotherapy in patients with small cancers of the breast. N Engl J Med 1981; 305:6-11.

2. Fisher B, Readmond C, Poisson R, et al. Eight-year result of a randomized clinical trial comparing total mastectomy and lumpectomy with or without radiation in the treatment of breast cancer. $\mathrm{N}$ Engl J Med 1989; 320:822-8.

3. Early stage breast cancer. Consens Statement 1990 Jun 18-21; 8:1-19.

4. Kurtz JM, Kinkel K. Breast conservation in the $21^{\text {st }}$ century. Eur J Cancer 2000; 36:1919-24.

5. Pinotti JA, Carvalho FM. Intraoperative pathological monitorization of surgical margins: a method to reduce recurrences after conservative treatment breast cancer. Eur J Gynaecol Oncol 2002; 23:11-6.

6. Stavros AT, Thickman D, Rapp CL, Dennis MA, Parker SH, Sisney GA. Solid breast nodules: use of sonography to distinguish between benign and malignant lesions. Radiology 1995; 196:123-34.

7. Berg JW. The significance of axillary node levels in the study of breast carcinoma. Cancer 1955; 8:776-8.

8. International Commission on Radiological Protection. A report on progress towards new recommendations: a communication from the International Commission on Radiological Protection. J Radiol Prot 2001; 21:112-23.

9. Ministério da Saúde. Conselho Nacional de Saúde. Normas de pesquisa envolvendo seres humanos Res. CNS 196/96. Bioética 1996; 4 Supl:15-25.

10. Conselho Federal de Medicina. Código de ética médica. Brasília: O Conselho; 1988.
11.Veronesi U, Volterrani F, Luini A, et al. Guadrantectomy versus lumpectomy for small size breast cancer. Eur J Cancer 1990; 26:671-3.

12. Kurtz JM, Jacquemier J, Brandone H, et al. Inoperable recurrence after breast-conserving surgical treatment and radiotherapy. Surg Gynecol Obstet 1991 ; 172:357-61.

13. Arcangeli G, Micheli A, D'Angelo L, et al. Conservative surgery and radiotherapy in early stage breast cancer: a comparison between tumourectomy and quadrantectomy. Radiother Oncol 1998; 46:39-45.

14.Gage I, Schnitt SJ, Recht A, et al. Skin recurrences after breast-conserving therapy for early-stage breast cancer. J Clin Oncol 1998; 16:480-6.

15.Fisher ER, Sass R, Fisher B, Gregorio R, Brown R, Wickerham L. Pathologic findings from the National Surgical Adjuvant Breast Project (Protocol 6). II. Relation of local breast recurrence to multicentricity. Cancer 1986; 57:1717-24.

16.Singletary SE, Kroll SS. Skin-sparing mastectomy with immediate breast reconstruction. Adv Surg 1996; 30:39-52.

17.Slavin SA, Schnitt SJ, Duda RB, et al. Skin-sparing mastectomy and immediate reconstruction: oncologic risks and aesthetic results in patients with early-stage breast cancer. Plast Reconstr Surg 1998; 102:49-62.

18.Toth BA, Forley BG, Calábria R. Restrospective study of the skin-sparing mastectomy in breast reconstruction. Plast Reconstr Surg 1999; 104:77-84.

19.Simmons RM, Fish SK, Gayle L, et al. Local and distant recurrence rates in skin-sparing mastectomies compared with non-skin-sparing mastectomies. Ann Surg Oncol 1999; 6:676-81.

20. Hidalgo DA. Aesthetic refinement in breast reconstruction: complete skin-sparing mastectomy with autogenous tissue transfer. Plast Reconstr Surg 1998; 102:63-70.

21.Peyser PM, Abel JA, Straker VF, Hall VL, Rainsbury RM. Ultra-conservative skin-sparing "keyhole" mastectomy and immediate breast and areola reconstruction. Ann R Coll Surg Engl 2000; 82:227-35.

22.Kroll SS, Ames F, Singletary SE, Schusterman MA. The oncologic risks of skin preservation at mastectomy when combined with immediate reconstruction of the breast. Surg Gynecol Obstet 1991; 172:17-20.

23.Barros AC, Teixeira LC, Nisida AC, Pinotti M, Pinotti JA. Prognostic effects of local recurrence after conservative treatment for early infiltrating breast carcinoma. Tumori 2002; 88:376-8.

Recebido em: 27/7/2003 Aceito com modificações em: 8/8/2003 